\title{
Error assessment of satellite-derived lead fraction in the Arctic
}

\author{
Natalia Ivanova, Pierre Rampal, and Sylvain Bouillon \\ Nansen Environmental and Remote Sensing Center, and Bjerknes Centre for Climate Research, Bergen, Norway \\ Correspondence to: Natalia Ivanova (natalia.ivanova@nersc.no)
}

Received: 1 October 2015 - Published in The Cryosphere Discuss.: 18 November 2015

Revised: 1 March 2016 - Accepted: 2 March 2016 - Published: 14 March 2016

\begin{abstract}
Leads within consolidated sea ice control heat exchange between the ocean and the atmosphere during winter, thus constituting an important climate parameter. These narrow elongated features occur when sea ice is fracturing under the action of wind and currents, reducing the local mechanical strength of the ice cover, which in turn impact the sea ice drift pattern. This creates a high demand for a highquality lead fraction (LF) data set for sea ice model evaluation, initialization, and for the assimilation of such data in regional models. In this context, an available LF data set retrieved from satellite passive microwave observations (Advanced Microwave Scanning Radiometer - Earth Observing System, AMSR-E) is of great value, which has been providing pan-Arctic light- and cloud-independent daily coverage since 2002. In this study errors in this data set are quantified using accurate LF estimates retrieved from Synthetic Aperture Radar (SAR) images employing a threshold technique. A consistent overestimation of LF by a factor of 2-4 is found in the AMSR-E LF product. It is shown that a simple adjustment of the upper tie point used in the method to estimate the LF can reduce the pixel-wise error by a factor of 2 on average. Applying such an adjustment to the full data set may thus significantly increase the quality and value of the original data set.
\end{abstract}

\section{Introduction}

In winter leads control heat transfer between the ocean and the atmosphere despite their relatively small areal coverage. For instance, sensible heat flux through leads can be of the order of $600 \mathrm{~W} \mathrm{~m}^{-2}$, compared to an annual average of about $3 \mathrm{~W} \mathrm{~m}^{-2}$ over ice (Maykut, 1978). This applies to leads represented by both open water and thin ice, but in winter, the refreezing happens very quickly and open water leads ex- ist only for a very short time (Weeks, 2010). Open-water leads alone, even though covering only $1-2 \%$ of the central Arctic, contribute more than $70 \%$ to the upward heat fluxes (Marcq and Weiss, 2012). Model simulations showed that even $1 \%$ change in sea ice concentration due to the increase in areal lead fraction could lead to a $3.5 \mathrm{~K}$ difference in the near-surface atmospheric temperature (Lüpkes et al., 2008). Studying signatures of leads and surrounding ice in the images from Moderate Resolution Imaging Spectroradiometer (MODIS), Beitsch et al. (2014) showed that the difference in ice surface temperature between thicker ice and a lead covered by thin ice could be as large as $15-20 \mathrm{~K}$, while open water and thin ice in leads differed in temperature by up to $10 \mathrm{~K}$ (Fig. 2 in Beitsch et al., 2014). This makes the surface energy budget very sensitive to the fraction of the surface covered by leads in the Arctic, where in recent years sea ice cover has become younger (Maslanik et al., 2007) and mechanically weaker (Rampal et al., 2009).

Areal fraction of leads in the Arctic sea ice can be viewed as a parameter reflecting loss in mechanical strength of the ice pack and indicating the degree of surrounding sea ice mobility. Rampal et al. (2009) reported a steady increase in sea ice deformation rate and drift during 1979-2007 and argued for possible causal relation between the two. These trends still remain a challenge to capture for the current sea ice models, especially because they fail at simulating sea ice fracturing and lead opening with the correct properties. Accurate observations of lead fraction are thus of high importance for model evaluation and for being assimilated into models as initial conditions, or during a simulation. For example, Bouillon and Rampal (2015) and Rampal et al. (2015) recently presented a new sea ice model, which is able to use information on lead fraction to constrain the local mechanical response of sea ice to winds and currents, with a significant impact on performance with respect to e.g. simulated sea ice drift 
and deformation. In this context, using accurate estimates of lead fraction with their associated uncertainties is therefore crucial.

A method for areal lead fraction (LF) retrieval from Advanced Microwave Scanning Radiometer - Earth Observing System (AMSR-E) was developed by Röhrs and Kaleschke (2012) (see also Röhrs et al., 2012) and allows leads wider than $3 \mathrm{~km}$ to be detected. The method was able to detect $50 \%$ of leads when compared to a MODIS image and localize the leads correctly when qualitatively compared to Synthetic Aperture Radar (SAR) images and CryoSat2 tracks (Röhrs et al., 2012). A daily light- and cloudindependent pan-Arctic LF data set (AMSR-E LF) for winter months November-April from 2002 to 2011 was obtained using this method and published at Integrated Climate Date Center - ICDC, University of Hamburg (http://icdc.zmaw. $\mathrm{de} /$ ), and represents a unique and valuable data set. It was then used to automatically obtain lead location and orientation with a success rate of $57 \%$ (Bröhan and Kaleschke, 2014). Preferred lead orientations were found typical for different regions of the Arctic.

The AMSR-E LF method is essentially a thin ice concentration retrieval method, which was adapted to identify leads by using median filtering. This filtering enhances the leads' features due to their narrow and elongated shape. Therefore, other thin ice retrieval methods based on passive microwave observations (e.g. Mäkynen and Similä, 2015; Naoki et al., 2008; Cavalieri, 1994) cannot be used directly for LF retrieval. A sea ice concentration algorithm, ASI (ARTIST sea ice; Svendsen et al., 1987; Kaleschke et al., 2001; Spreen et al., 2008), was able to identify leads (Beitsch et al., 2014) when implemented at $89 \mathrm{GHz}$ frequency of AMSR2 onboard the Global Change Observation Mission-Water satellite with a resolution of $3.125 \mathrm{~km}$. However, this approach is limited in time coverage because AMSR2 only started to deliver the data in 2012 (http://suzaku.eorc.jaxa.jp). Also, quantitative validation work may be still needed because only qualitative assessment using MODIS images was presented in Beitsch et al. (2014).

A lead detection method based on MODIS ice surface temperature was developed by Willmes and Heinemann (2015). The method classifies a scene into leads and artefacts, where for the first class (leads) the success rate is as large as $95 \%$. However, in the class of artefacts, which are mostly caused by ambiguity in cloud identification, there is a $50 \%$ chance of it being either a lead or an artefact. Combined retrieval error from the two classes for a daily map, obtained by averaging, is estimated to be $28 \%$. The method gives daily lead occurrence maps at $1 \mathrm{~km}^{2}$ resolution.

A number of classifiers applied to CryoSat-2 were tested for lead detection potential, and the most promising one identified and used to derive LF and lead width distribution (Wernecke and Kaleschke, 2015). The selected classifier was able to detect $\sim 68 \%$ of leads correctly, and only $\sim 3 \%$ of ice measurements were falsely identified as leads.
Despite such good capability and fine resolution of $250 \mathrm{~m}$, LF retrievals from CryoSat- 2 are limited spatially, because the measurements are conducted by tracks making daily panArctic coverage impossible, and temporally, the satellite being launched in 2010. Suggested approaches using laser altimeter for lead detection (e.g. Farrell et al., 2009 with the Ice, Cloud and land Elevation Satellite, ICESat) have similar limitations.

Lindsay and Rothrock (1995) suggested a method for retrieval of lead widths and LF from thermal and reflected solar channels on the Advanced Very High Resolution Radiometer (AVHRR). The nominal resolution of the instrument is $1.1 \mathrm{~km}$, and it is also able to resolve subpixel-sized leads due to strong contrast caused by leads and their network-like pattern. However, an AVHRR-retrieved LF data set would be limited to cloud-free areas, and its quality would depend on the quality of cloud masking defining these areas.

Automatic classification of leads from SAR is difficult, because radar backscatter signature of leads in SAR images can be ambiguous. This is due to wind roughening of the open water in the leads and occasional presence of frost flowers when new ice has just formed in a lead (Röhrs et al., 2012). To the authors' knowledge, no method has so far been presented in literature addressing automatic LF retrievals from SAR. Existing sea ice classification methods (Berg and Eriksson, 2012; Karvonen, 2012, 2014; Leigh et al., 2014; Liu et al., 2015) could potentially be adapted and tested for this purpose. However, the task of identifying such narrow elongated features as leads is different from sea ice classification. For example, Korosov et al. (2015b) demonstrated that these features could not be distinguished using a support vector machine (SVM) approach applied to SAR subsets (windows), though such a technique was good enough for ice/water separation in general. Also, this study showed that even applying this method to segments, which significantly improved its feature-resolving capacity, was not satisfactory, and that the SVM would need to be trained specifically to target leads.

As it is outlined above, there are a variety of available promising methods to detect leads and retrieve LF from satellites. They all have their advantages and disadvantages and, depending on these, can be used for achieving different purposes. The topic of this study is a data set meeting the following criteria: LF is retrieved (note the difference with lead occurrence); coverage is daily; data are pan-Arctic, cloud-, and light-independent; and data cover a longest possible time period. The AMSR-E LF appears to be the only suitable data set in this context, and therefore we find it necessary to provide quantitative error estimations of this data set, which has not been done before. Based on analysis of the errors, we suggest a possible improvement of the AMSR-E-based method. In order to achieve the goal of this study, a simple method for LF retrieval from SAR is suggested. Currently the method is specifically adapted for the purposes of this study, but fur- 
ther development can give a universal approach for areal LF retrieval from SAR, which would be highly valuable.

Following the Introduction, Sect. 2 of the paper describes the data used for the study, and Sect. 3 explains the SARbased method. The results are presented in Sects. 4 and 5 followed by Discussion and Conclusions.

\section{Data}

\subsection{The AMSR-E LF data set}

The daily gridded AMSR-E LF data set for the time period of November 2003-April 2011 was used (downloaded in February 2015, http://icdc.zmaw.de/1/daten/cryosphere/ lead-area-fraction-amsre.html). It covers winter months of November through April and is provided on a polarstereographic grid with $6.25 \mathrm{~km}$ resolution distributed by $\mathrm{Na}$ tional Snow and Ice Data Center (NSIDC). LF is expressed as the percentage of a grid cell covered by leads, which are represented by either open water or thin ice. Since openings refreeze very quickly in winter, the majority of the data entries are thin ice concentrations. Following the original paper of Röhrs et al. (2012), thin ice is defined as new ice, nilas, and pancake ice, according to the classification of the World Meteorological Organization (WMO, 1989). The data set is limited to areas where sea ice concentration is above $90 \%$, as retrieved by the ASI algorithm.

The method used to retrieve LF from AMSR-E (Röhrs et al., 2012) relies on the unique signature of thin ice and open water defined by brightness temperature ratio in the 89 and $19 \mathrm{GHz}$ vertically polarized channels of the radiometer. Further, median filtering is applied to exclude the part of the signal which comes from the atmosphere and to enhance the features of leads due to their narrow and elongated shape which is so different from the more homogeneous background.

The AMSR-E LF data set is shown in Fig. 1 by the number of measurements in each bin expressed in percent of the total number of measurements (relative frequency), where each bin has a width of $5 \%$ except the first one, which excludes LF $<1 \%$. These very small values of LF in the data set appeared rather random on the daily maps and therefore were excluded, assuming the method's precision would not have allowed them to be resolved anyway. All the grid cells close to land were also removed (two grid cells away from land) because these areas contained a large number of near $100 \%$ LF values, which may be caused by either real presence of the coastal polynyas/leads or an artefact due to the vicinity of land. Figure 1 shows the full data set covering all the winters from November 2003 through to April 2011 ( 26 million measurements) by blue bars, and each month from November 2008 to April 2009 (varying from 430 to $\sim 600$ thousand measurements) by different colours. The histogram for these months reflects the tendency observed in the full data set, thus allowing us to limit the analyses presented

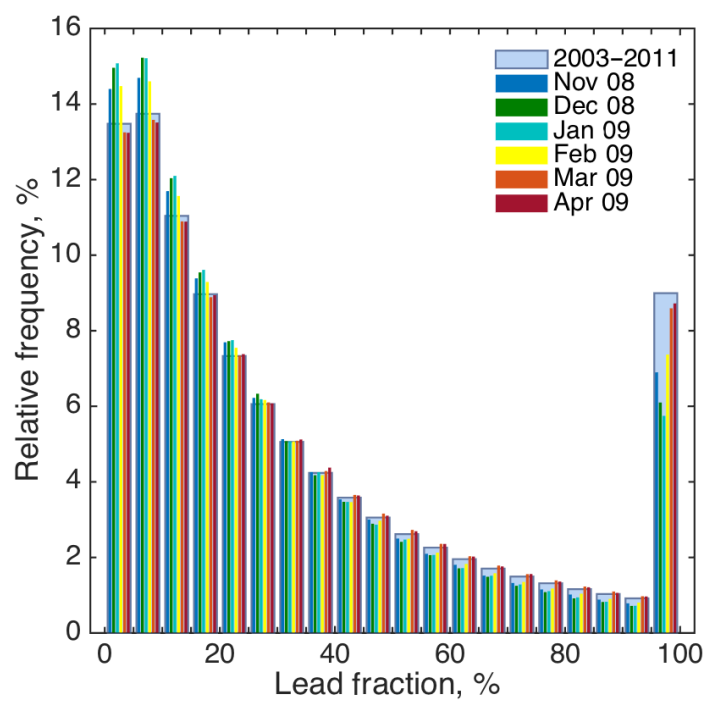

Figure 1. Histogram for the AMSR-E lead fraction (LF) data set shown as the number of measurements per each LF bin of $5 \%$ width expressed in percent of the total number of measurements (relative frequency). The blue bars show the full data set, while each month of the winter 2008-2009 is shown by other colours (see the legend).

in this paper to only this one winter. The last bin (LF 95$100 \%$ ), characterized by a significant number of measurements in comparison to the other bins with high LF values, will be addressed in later sections.

For the validation by SAR images the AMSR-E LF data set was reprojected on the domain defined in Sect. 2.2 using Nansat - an open-source Python toolbox for processing 2-D satellite earth observation data (Korosov et al., 2015a, 2016).

\subsection{The SAR images}

ENVISAT ASAR WSM (advanced SAR wide swath mode) images at $\mathrm{HH}$ (horizontal transmitting, horizontal receiving) polarization acquired during the winter of November 2008April 2009 were used in this study. The area of interest is defined by the geographical coordinates $\left(83^{\circ} \mathrm{N}, 20^{\circ} \mathrm{W}\right)$, $\left(87^{\circ} \mathrm{N}, 36^{\circ} \mathrm{W}\right),\left(87^{\circ} \mathrm{N}, 34^{\circ} \mathrm{E}\right),\left(83^{\circ} \mathrm{N}, 15^{\circ} \mathrm{E}\right)$ and is shown in Fig. 2 by the red rectangle. This area located north of the Fram Strait was chosen due to a relatively large number of leads occurring in this particular region (see e.g. Bröhan and Kaleschke, 2014) so that a sufficient number of AMSR-E LF retrievals would be available for validation, and because this region is well covered by SAR data. The SAR images originally provided at a spatial resolution of $150 \mathrm{~m} \times 150 \mathrm{~m}$ (pixel spacing: $75 \mathrm{~m} \times 75 \mathrm{~m}$ ), were reprojected using the Nansat toolbox onto a polar stereographic projection with a nominal resolution of $100 \mathrm{~m} \times 100 \mathrm{~m}$ with latitude of origin and central meridian defined by the central coordinates of the selected area. The calibrated surface backscattering coefficient (ASAR Product Handbook, 2007) normalized over ice was used for this study (we will refer to this value as backscatter). 


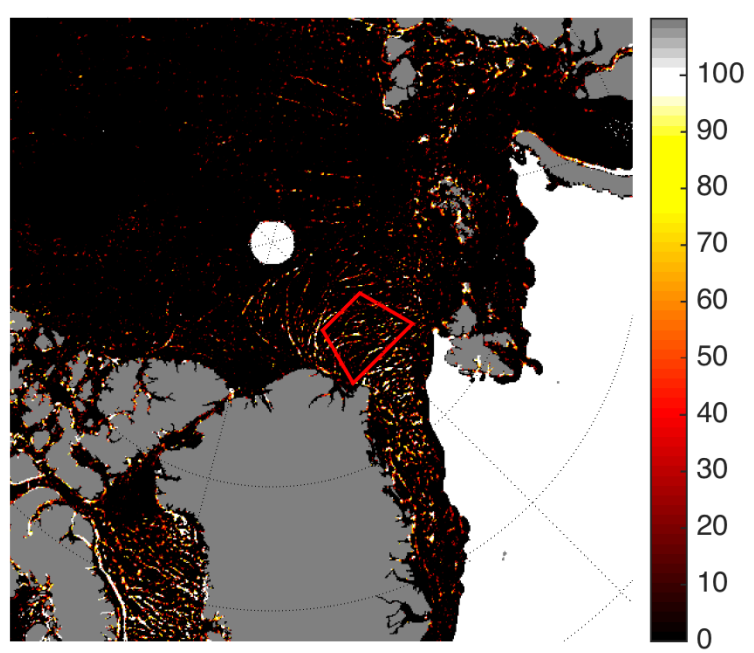

Figure 2. Area of interest is included within the red rectangle. The background map shows AMSR-E lead fraction in percent (the numbers on the colour scale to the right), obtained on the 8 March 2009, and is used here only to demonstrate a sample from the product.

The procedure of normalization represents compensation for incidence angle variation, established empirically, and is described in more detail in Zakhvatkina et al. (2013).

\section{SAR-based threshold technique}

A threshold technique similar to the one developed for lead detection from MODIS-derived ice surface temperature (Willmes and Heinemann, 2015) is suggested for automatic lead identification in SAR scenes. Visual inspection of SAR images shows that leads, in most cases, have lower backscatter than surrounding thicker ice. The transition is defined by a threshold, which is not constant from one image to another, as we find from automatic lead detection tests conducted on a number of SAR images. Therefore, we use characteristics of backscatter distributions for each SAR scene instead. Before the threshold can be applied to a SAR scene (a subset is shown in Fig. 3a and respective distribution in Fig. 3d, beige bars) the image undergoes median filtering with a window size of $5 \times 5$ pixels (found experimentally), corresponding to a spatial scale of $500 \mathrm{~m} \times 500 \mathrm{~m}$, which reduces the noise while preserving the edges of the features. One such filtered subset of a SAR image is shown in Fig. 3b (distribution in Fig. 3d, blue bars), where dark blue areas correspond to leads. Comparison of distributions before filtering (wider) and after shows the noise-reducing effect of the median filtering. After applying the threshold, so that all the backscatter values below its value are classified as leads - and the rest as ice - a binary map (Fig. 3c) is retrieved. The threshold $\left(\sigma_{0}^{t}\right)$ is defined as

$\sigma_{0}^{t}=\sigma_{0}^{\mathrm{P}}-n_{\delta} \cdot \delta$,
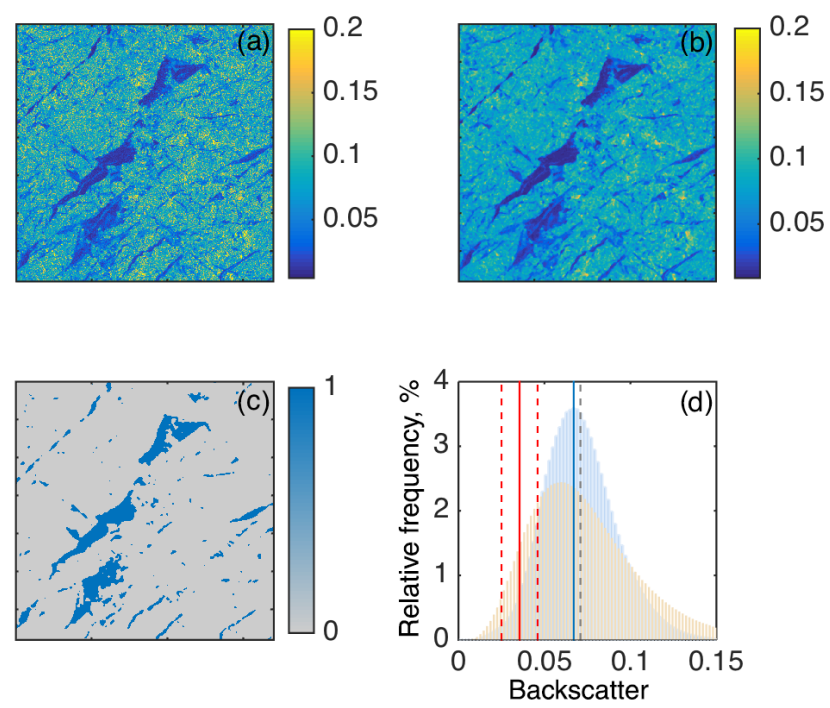

Figure 3. Threshold technique used to calculate lead fraction from SAR images: (a) a subset of $680 \times 680$ pixels showing backscatter values; (b) same as (a) but after median filter has been applied; (c) the resulting lead detection (1 - lead, 0 - ice); (d) histogram of an example SAR scene taken on the 1 March 2009 (blue) with lines showing the peak (blue), threshold defined as peak minus 1.5 standard deviation (red), other thresholds (when 1 standard deviation and 2 standard deviations are used, dashed red); and the mean is shown by the grey dashed line. The beige bars represent the unfiltered signal.

where $\sigma_{0}^{\mathrm{P}}$ is the backscatter value at the peak of the distribution (blue line in Fig. 3d), $\delta$ is the standard deviation of the distribution, and $n_{\delta}$ is a number of standard deviations to move away from the peak, that enables automatic identification of leads. The threshold was first tried with $n_{\delta}=1$ and $n_{\delta}=2$ (dashed red lines), but it was found that an intermediate value $n_{\delta}=1.5$ (solid red line) worked better and was therefore chosen. This was established by visual comparison of the lead fraction retrievals with different threshold values. The mean of the distribution is shown by a dashed grey line for reference.

Next, the SAR-based LF is calculated for each AMSRE grid cell where the LF value is above $1 \%$. All the pixels classified as lead by SAR within such a grid cell are added together and divided by the total number of SAR pixels in it, which gives a percentage after it is multiplied by 100 .

The suggested approach is rather simplistic, but it is sufficient for our purpose (more details in Sect. 4.1.2 and 4.3), while for a wider application, one must consider the limitations addressed in the Discussion section. 


\section{Results}

\subsection{Reference lead fraction data sets retrieved from SAR}

Using the approach described in Sect. 3, we produced two SAR-based reference data sets: one with manual quality control of each SAR subset of $1000 \times 1000$ pixels (manual quality control (MQC) SAR LF), and one based on an automatic threshold where quality control is done by discarding images with obviously unsuccessful LF retrievals (SAR LF).

\subsubsection{MQC SAR LF}

This high-quality data set was produced in order to verify the larger SAR LF data set (Sect. 4.1.2). A significantly larger number of measurements in the SAR LF allows robust statistical analysis, but visual quality control of each image, given that leads are numerous small features, is hardly achievable. For the MQC SAR LF, two criteria need to be verified: (1) whether the classification is successful and (2) whether leads are identified in exactly the same locations in the SAR- and AMSR-E-derived data sets. The latter was mostly the case; however, sometimes a lead in AMSR-E LF was misplaced by a distance large enough to cause the two data sets to mismatch. We believe this misplacement is caused by cases of relatively fast sea ice drift in the area. If we consider an AMSR-E grid cell of $6.25 \mathrm{~km} \times 6.25 \mathrm{~km}$ size, a SAR image is taken at a certain time of the day in this grid cell, while ASMR-E LF is a gridded daily product and thus provides an average over all the swaths covering this grid cell collected during $24 \mathrm{~h}$. During a few hours the lead could have moved fast enough to disappear from the given grid cell. From visual analysis of the images we could say that this situation did not happen very often; however, a quantitative estimate of how much it affects the validation was needed. Thus, we make an assumption that if the distribution of SAR LF is similar to that of MQC SAR LF, where we made sure every lead was located correctly, the misplacements were indeed seldom the case also in the SAR LF data set.

To produce the MQC SAR LF, five SAR scenes acquired in March 2009 with a sufficient number of easily distinguishable leads were selected. It was found that the quality of LF retrieval increases when dividing SAR scenes into subsets, and the subset size of $1000 \times 1000$ pixels was shown to be sufficient. Using such small subsets rather than a full SAR image provides more accurate thresholds because it limits possible variability in conditions within the subset. Such conditions can be wind speed or ice surface properties (wet or dry ice, for example). Defining a threshold locally not only eliminates significance of these effects, but it also takes advantage of a smaller variety of surfaces in general. For example, presence of open water, land, consolidated ice, wet ice, dry ice, and marginal ice zone in one image will make it difficult to find a threshold that will only identify leads. Using a smaller subset, on the other hand, where only consolidated ice with leads is present, will give a clearer threshold.

The threshold was thus calculated individually for each $1000 \times 1000$ pixels subset using Eq. (1) with $n_{\delta}$ selected manually, and used to calculate LF in corresponding AMSR$\mathrm{E}$ grid cells. The classification in each subset was then inspected visually, comparing the two collocated maps, backscatter and MQC SAR LF, in order to make sure it was successful. This procedure gave 1645 high-quality MQC SAR LF retrievals, which were then used to verify the findings based on a larger SAR LF data set.

\subsubsection{SAR LF}

To produce this data set, SAR subsets of $3500 \times 3500$ pixels each (on average) were used: the full SAR images were cut to match the region of interest (Fig. 2). The quality control of this validation data set was done by visual inspection of every classified subset together with the original SAR subset (backscatter) as demonstrated in Fig. 4 (a subset of $875 \times 875$ pixels is shown). Panel a shows the original SAR image; panel $b$ shows the lead identification by the SARbased method, where the red colour corresponds to the identified leads; and panel c shows the SAR image overlaid by the AMSR-E lead fraction original product (in \%) with the colour scale to the right. We rely on the combination of the fine resolution of SAR (pixel spacing of original product: $75 \mathrm{~m} \times 75 \mathrm{~m}$, geometric resolution: $150 \mathrm{~m} \times 150 \mathrm{~m}$ ) and its capability to separate smooth surfaces such as open water or thin ice in leads (appear darker than the background) from the rough surfaces (surrounding thicker ice). In addition, leads have a characteristic shape: they are narrow elongated features. These three factors put together make it possible to visually recognize the leads in SAR images. The features that were missed by SAR were relatively small, and were usually not captured by the coarse-resolution AMSR-E either (Fig. 4c). Note also that the AMSR-E-based method was found to identify only leads wider than $3 \mathrm{~km}$ (Röhrs et al., 2012), and such features were normally identified successfully by SAR in our study. We also performed a visual comparison of a SAR data set sample to a MODIS image $(2500 \times 2500$ pixels at $250 \mathrm{~m}$ resolution $)$, and saw that the SAR method captured the majority of features correctly.

In this process, images were discarded in cases of unsuccessful lead identification, which is when a significant number of features that appear like leads were missed by the method. This was of particular importance in cases when AMSR-E LF identified a feature in the respective location, to secure proper error estimation for the AMSR-E LF product.

The majority of subsets contained leads represented by signatures darker than the surrounding background, while subsets containing a large number of leads with brighter signature were discarded. This means that the majority of the leads in the selected subsets were either composed of thin ice 


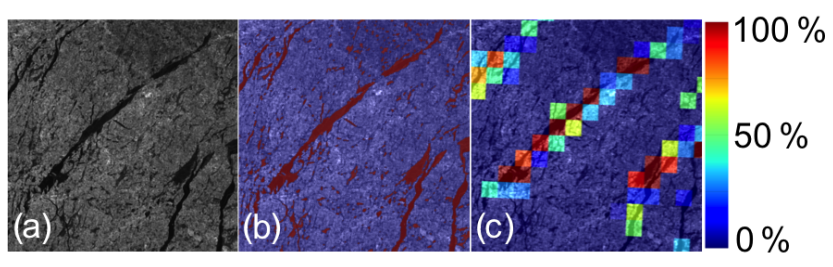

Figure 4. (a) A subset of the original SAR image (backscatter) on the 8 March 2009; (b) respective classified SAR image (red - lead, blue - ice) with (a) as background; (c) AMSR-E lead fraction in percent with (a) as background.

Table 1. Number of measurements in the SAR LF data set.

\begin{tabular}{llr}
\hline Month & Subsets & Measurements \\
\hline Nov 2008 & 27 & 8097 \\
Dec 2008 & 34 & 9392 \\
Jan 2009 & 47 & 10672 \\
Feb 2009 & 29 & 7528 \\
Mar 2009 & 47 & 19460 \\
Apr 2009 & 21 & 8914 \\
\hline Total & 205 & 64063 \\
\hline
\end{tabular}

or calm open water. Therefore, the wind speed is not taken into account in this study, but for a more general application this would have been necessary to account for wind roughening of the open water areas in leads. As a result we obtained a data set for the period of November 2008-April 2009, made of $21-47$ subsets $(3500 \times 3500$ pixels each) per month, with the number of measurements varying from about 8000 to 19500 (Table 1) depending on the month.

\subsection{Comparison of the AMSR-E LF and MQC SAR LF}

Before any analysis of the AMSR-E LF and MQC SAR LF data sets could be performed, they were filtered so that only those AMSR-E grid cells $(6.25 \mathrm{~km}$ by $6.25 \mathrm{~km}$ size) were used, which had an LF value $>1 \%$ and where the SAR LF for this grid cell returned a value of $L F>1 \%$ too. Thus, we only analyse the non-zero values of the AMSR-E LF data set, and exclude all the leads that the SAR method eventually missed. The same applies to the next section, where AMSRE LF is compared to SAR LF.

The AMSR-E LF and MQC SAR LF data sets are shown in Fig. 5 as a scatter plot (left) and histogram (right). The scatter plot shows that the majority of the points are located below the 1-to-1 line (dashed grey line), which means that in most cases AMSR-E LF overestimates the LF as compared to the SAR retrievals. The linear regression line (red) has a slope of 0.2. Note that for the value of AMSR-E LF $100 \%$, there is a wide range of MQC SAR LF values covering almost the full scale from 0 to $100 \%$. The point-wise root mean square error,
$\operatorname{RMSE}=\sqrt{\frac{1}{n} \sum_{i=1}^{n}\left(\operatorname{LF}_{\mathrm{AMSRE}} i-\mathrm{LF}_{\mathrm{SAR}} i\right)^{2}}$,

where $n$ is the total number of measurements (1645 in this case), and is equal to $33 \%$. The determination coefficient, $R^{2}$, retrieved as a squared correlation coefficient and expressed in percent, is $13 \%$ (significant). The right panel of Fig. 5 shows a histogram of the two data sets representing the number of measurements per each $5 \%$ bin expressed in percent of the total number of measurements. The distributions of the two data sets look principally different, characterized by a steep decrease in the number of cases with increasing LF for SAR and a wide distribution of values in the AMSR LF. Thus, for LF $>20 \%$ AMSR LF seems to largely overestimate the number of cases and underestimate this number for lower LF values. Similar to the full AMSR-E LF data set (Fig. 1.), the near $100 \%$ bin contains a relatively large number of measurements. In fact, about $94 \%$ of all the data in this bin in the full AMSR-E data set are above $99.9 \%$. In order to understand the origin of such a large amount of LF near $100 \%$, we compare spatial maps of LF obtained from AMSR-E and SAR. As an example of such analysis, Fig. 6 shows part of a SAR image overlaid by the collocated AMSR-E LF product, where one can see general overestimation of LF by AMSR-E (larger grid cells shown as a percentage by different colours). In particular, it is clear for the LF $100 \%$ cases (red grid cells) that these often correspond to a smaller amount of water/thin ice in the SAR image. Four neighbouring AMSR-E grid cells are shown in a close-up inset, where three of them have a LF value of $100 \%$ (the fourth one has no value), while the SAR image in the background clearly contains one lead that covers only about $25 \%$ of the right grid cell, $40 \%$ of the upper grid cell, and about $60 \%$ of the left one, where also smaller cracks are present.

\subsection{Error estimations of the AMSR-E LF based on SAR LF}

The same procedure as in Sect. 4.2 is now applied using the large SAR LF data set. Histograms for collocated data sets AMSR-E LF and SAR LF were produced for each month of the considered period (Fig. 7). They show the same tendency as when using the shorter high-quality data set. The distributions here are much smoother because of the significantly larger number of measurements. The similarity of the distributions coming from high-quality MQC SAR LF and SAR LF allow us to base our conclusions on the larger data set (SAR LF) thus providing more accurate estimates of errors.

With this significant number of collocated SAR and AMSR-E retrievals of LF, we can confirm that the peak in AMSR-E LF data set near $100 \%$ represents an artefact. This is also supported by the visual analysis of overlay of every image pair, AMSR-E LF and SAR LF. AMSR-E LF had 

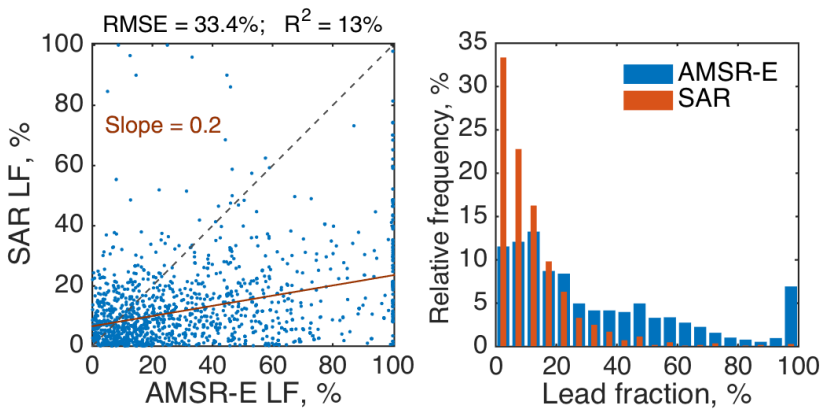

Figure 5. A comparison of the AMSR-E lead fraction (LF) and SAR LF with manual quality control (MQC); the total number of measurements is 1645. Left: scatter plot of MQC SAR LF vs. AMSR-E LF (\%). The 1-to-1 line is the dashed grey line, and the linear regression is shown by the red line and the slope value. The root mean square error (RMSE) and the coefficient of determination $\left(R^{2}\right)$ are shown at the top of the plot. Right: histogram for the two data sets shown as a percentage of measurements per each bin of $5 \%$ width (relative frequency).

a relatively large number of observations close to $100 \%$, while in SAR images, the area covered by leads in such grid cells was obviously smaller in almost all the cases. The cases where one lead width would take the full AMSR-E grid cell or even more (lead width larger than one grid cell) were extremely rare in our selection. We believe that this grouping of a large number of measurements near the value of $100 \%$ is a result of the assumption lying behind the AMSR-E method for LF retrieval. The method is based on the ratio of the brightness temperatures $(r)$ in 89 and $19 \mathrm{GHz}$ channels (Röhrs et al., 2012). The assumption is that all the values of this ratio above a certain constant value (a tie point) will give LF $100 \%$. All the other values are linearly interpolated between a tie point for LF $0 \%(r 0)$ and a tie point for LF $100 \%(r 100)$. If the upper tie point $r 100$ is too low, a significant number of LF values assigned to a value of $100 \%$ by this cut-off may actually correspond to a variety of LF much lower than $100 \%$. This is reflected in Fig. 5 (left) and Fig. 6, where values of LF $100 \%$ in the AMSR-E data set correspond to a variety of values from the SAR data set. Ideally, an improvement of the ASMR-E LF method is needed, for example, by adjusting the upper tie point so that the full range of LF values are covered. We address this further in Sect. 5 .

Since production of a new improved AMSR-E LF data set is outside of the scope of this study, we suggest imitating the same problem with the SAR LF data set instead. The introduction of a new upper tie point $r^{\prime} 100$ would be equivalent to dividing all the AMSR-E LF values by a certain factor, defined as $f=\left(r^{\prime} 100-r 0\right) /(r 100-r 0)$, because the method is based on linear interpolation of all the values between the limits of the range. Since the LF values in the near $100 \%$ bin for AMSR LF are unknown, we suggest multiplying the SAR LF data set by such a factor instead. In order to define

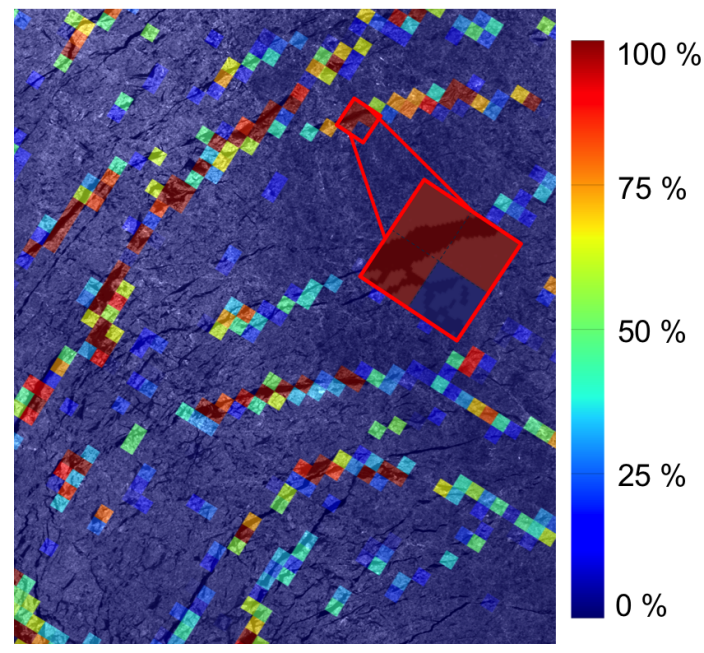

Figure 6. Subset of a SAR image taken on the 8 March 2009 overlaid by the collocated AMSR-E lead fraction (LF) product, where red grid cells correspond to LF $100 \%$ (for the other values, see the colour scale on the right). The zoomed-in inset shows four grid cells of which three have AMSR-E LF $100 \%$ and one has LF $0 \%$.
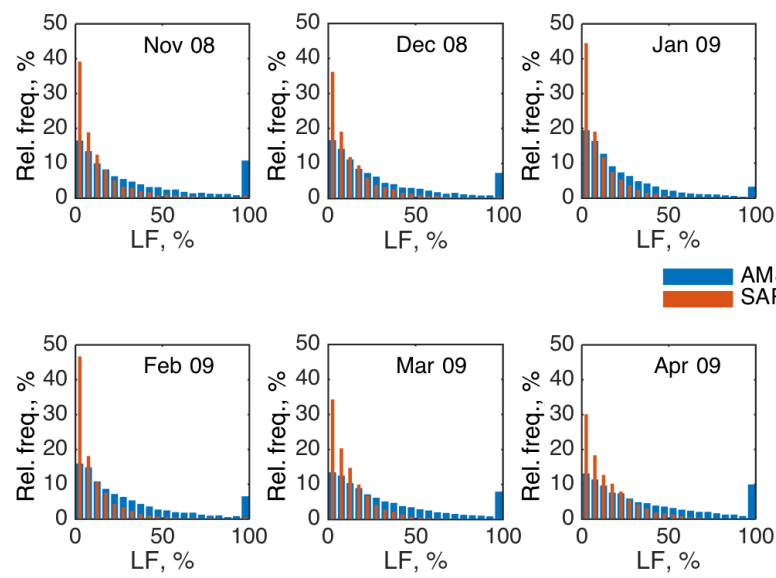

Figure 7. Histograms of the AMSR-E lead fraction (LF) and SAR LF data sets for every month from November 2008 to April 2009 shown as a percentage of measurements per each bin of $5 \%$ width (relative frequency). The total number of measurements amounts to 64063 in 205 subsets size of $3500 \times 3500$ pixels each, and is given for each month in Table 1 .

the value of $f$ (also referred to as AMSR-E factor) we vary its value from 1 to 5 and calculate the respective RMSE as a measure of difference between the histograms of AMSR-E LF and SAR LF data sets for each month (Fig. 7.):

$\operatorname{RMSE}_{h}=\sqrt{\frac{1}{n_{\mathrm{b}}} \sum_{i=1}^{n_{\mathrm{b}}}\left(\operatorname{RF}_{\mathrm{AMSRE} i}-\mathrm{RF}_{\mathrm{SAR} i}\right)^{2}}$,

where RF stands for relative frequency in each bin, and $n_{\mathrm{b}}$ is the number of bins. The obtained $\mathrm{RMSE}_{h}$ is plotted as a function of $f$ in Fig. 8 (left), where each month is assigned a 

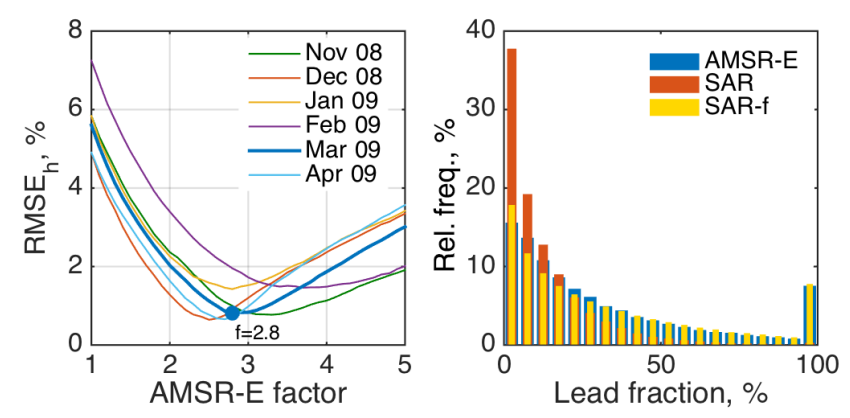

Figure 8. Left: Root mean square error $\left(\mathrm{RMSE}_{h}, \%\right.$, Eq. 3) as a measure of difference between the histogram of AMSR-E lead fraction (LF) and SAR LF multiplied by different values of $f$ (AMSR-E factor). To demonstrate the principle, March 2009 is highlighted by a bold blue line with a minimum factor of 2.8. Right: original histogram of AMSR-E LF and SAR LF data for the full winter November 2008-April 2009, and SAR LF multiplied by the respective factor for each month (yellow bars).

different colour and March 2009 is highlighted by a bold line to illustrate the principle. By minimizing $\mathrm{RMSE}_{h}$ we find an optimal $f$ value for each month, which amounts to $3.3,2.5$, 2.8, 3.7, 2.8, and 2.7 for the months from November 2008 to April 2009 respectively. Multiplying the SAR LF data set for each month by the respective factor gives a histogram with a similar issue at $100 \%$ as the AMSR-E LF data set has (high relative frequency in the last bin; yellow bars in Fig. 8, right). The values in other bins are also redistributed in a way that is similar to the AMSR-E LF data set. The original histogram of AMSR-E LF and SAR LF (same as Fig. 7, but for the full winter) data is also shown for reference.

The systematic overestimation of AMSR-E LF data also affects the mean value of the distribution. For winter 2009, the mean value of AMSRE LF ( $\overline{\mathrm{LF}_{\mathrm{AMSRE}}}$ ) is equal to $31 \%$, whereas it is equal to $13 \%$ for the SAR LF $\left(\overline{\mathrm{LF}_{\mathrm{SAR}}}\right)$. The absolute relative difference $100 \times$ $\left|\left(\overline{\mathrm{LF}_{\mathrm{AMSRE}}}-\overline{\mathrm{LF}_{\mathrm{SAR}}}\right) / \overline{\mathrm{LF}_{\mathrm{SAR}}}\right|$. decreases from $140 \%$ with no correction to $17 \%$ when using the correction factors found here.

Finally the agreement between SAR LF and AMSR-E LF data sets can be estimated by the point-wise RMSE of LF for the whole winter 2009 as defined by Eq. (2), the total number of measurements $n$ being 64063 here. Here $\mathrm{LF}_{\mathrm{SAR} ; i}$ are the LF values obtained when multiplying by the correction factor, so that point-wise RMSE is relatively independent of the systematic bias in AMSR-E LF. The point-wise RMSE is equal to $43 \%$ and is an estimate of the standard deviation of the difference between AMSRE-E LF and SAR LF. However, the similar computation of RMSE using LF $_{\mathrm{SAR}} i$ without correction gives a value of $33 \%$, suggesting the need for a more physically justified approach, e.g. by improving the AMSR-E-based method.

\section{Suggested improvement of the AMSR-E-based method}

In Sect. 4.3 we made an assumption that the upper tie point in the AMSR-E-based method should be increased in order to cover the full range of LF values. To test this assumption we implement the method according to Röhrs et al. (2012) and calculate LF from the AMSR-E brightness temperatures on the 8 March 2009 with the original tie points (a subset is shown in Fig. 9, upper left), i.e. with the upper tie point $r 100=0.05$. Such calculations give a similar distribution of LF values (Fig. 9, upper right) as was found in the full AMSR-E LF data set (Fig. 1). Using the linear relationship between $r 100$ and $f$, and the optimal value of $f$ for March $2009(f=2.8)$, we calculate that $r 100$ should be increased to $0.113\left(r^{\prime} 100\right)$. This new tie point value gives a distribution closer to that of the SAR LF data set (Fig. 9, bottom right) - the value of $\mathrm{RMSE}_{h}$ (Eq. 3) decreasing from $5.4 \%$ (corresponding to $f=1$ in Fig. 8, left) to $0.9 \%$. The closeup insets similar to the one in Fig. 6 show that the leads are identified in the same locations as before, but the LF values are lower (Fig. 9, bottom left). Fig. 10 (left) shows a similar scatter plot to Fig. 5 (left), but for this 1-day example. The right panel of Fig. 10 compares the AMSR-E lead fraction obtained with the new tie point to that of the reference SAR LF data set. The tie-point adjustment made the AMSR$E$ and SAR data sets agree significantly better: the point-wise RMSE (Eq. 2) for this 1-day data set of 750 collocated LF measurements decreased from 37 to $15 \%$ and the slope of the regression line became closer to 1 (increased from 0.2 to 0.5 ). The determination coefficient, $R^{2}$, showed also slight improvement, increasing from 27 to $33 \%$ (both coefficients are significant).

We thus believe that implementation of such an adjustment to the full AMSR-E LF data set will lead to a much better agreement with the SAR LF data set. The new tie point $r^{\prime} 100$ retrieved for the other months amounts to 0.131 , $0.103,0.113,0.145$ for November 2008-February 2009 respectively, and 0.110 for April 2009. The average value of the new tie point $r^{\prime} 100$ weighted by the number of observations for each month is 0.117 and is therefore our best estimate for winter 2008-2009.

\section{Discussion}

A method to retrieve LF from the SAR backscattering coefficient is introduced. This simple threshold technique is only suitable for the purposes of this study, and is thus not universal. However, its potential is shown, and the limitations are identified, allowing further developments of such a method, which is outside of the scope of this study.

One of the limitations is the ambiguity of SAR signatures corresponding to leads. When a lead is represented by calm open water or thin ice, it has lower backscatter val- 

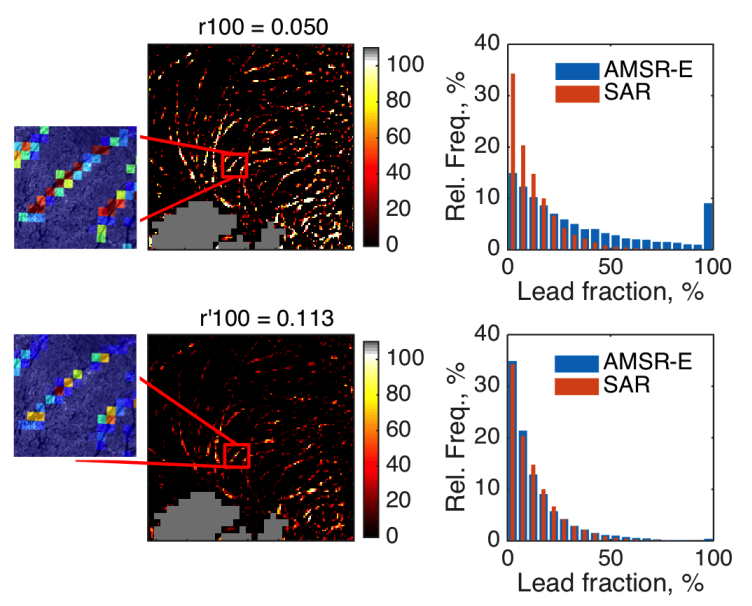

Figure 9. Adjustment of the upper tie point ( $r$ 100) of the AMSR-Ebased method. Upper panels: a subset of lead fraction (LF) values located in the area of interest (Fig. 2) (left) and distribution calculated from the full LF map (entire Arctic) on the 8 March 2009 (right, blue bars). The original $r 100$ value is used. The orange bars show the SAR LF distribution for the whole month of March 2009 for reference. Bottom panels: same, but for the adjusted $r^{\prime} 100$.
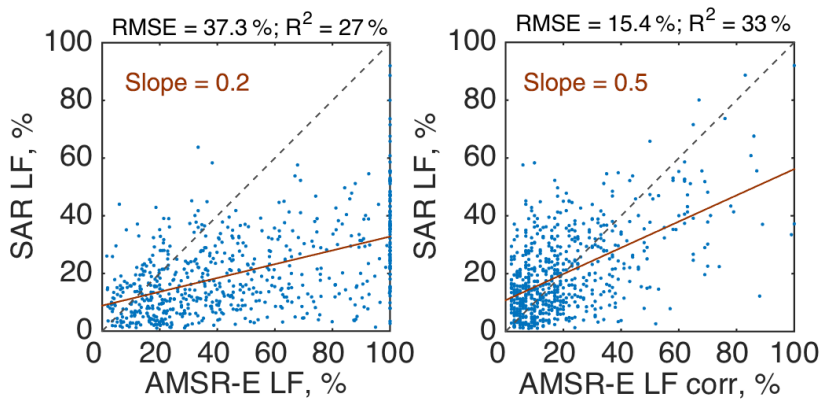

Figure 10. Left: scatter plot of SAR LF and AMSR-E LF using the original method of Röhrs et al. (2012). Right: same but using the adjusted AMSR-E method (new tie point). The 1-to-1 lines are the dashed grey lines, and the linear regressions are shown by the red lines and the values of the slope. The root mean square error (RMSE) and the coefficient of determination $\left(R^{2}\right)$ are shown at the top of the plots.

ues than surrounding thicker ice and therefore can be identified by a threshold. However, in cases when wind is roughening the open water surface in the lead, its signature becomes brighter. Another case of such ambiguity is the presence of frost flowers on the newly refrozen lead, which also causes brighter signatures (Röhrs et al., 2012). Such leads with brighter signature than the background are not identified by the presented SAR method, but are sometimes (but not always) identified by the AMSR-E method. These cases did not occur much in the considered examples and were discarded from the analysis, thus not affecting the conclusions. For a more universal SAR-based method such cases can be included by introducing two thresholds - one for the leads appearing darker than the background and one for the ones appearing brighter. In that case two different sides of the backscatter distribution will be used independently.

Another limitation of the approach used here is the presence of areas with presumably wet snow/ice, which appear rather dark on a SAR image and therefore are classified as leads by the threshold method. These cases did not occur often in our selection, and they did not influence the comparison because AMSR-E LF usually does not identify leads in such areas, and we had only included the grid cells where the AMSR-E LF data set had any values above $1 \%$. The threshold is also sensitive to the sea ice thickness. At a given threshold, only leads with ice thin enough will be identified as leads. Since we do not know how thick the ice is, it adds to the ambiguity of such a method. In other words, by selecting a threshold we indirectly set the sea ice thickness limit. When the distribution is bimodal (one mode for leads and one for thicker ice), a value between the peaks can be used as the threshold, as suggested by Lindsay and Rothrock (1995) for distributions of temperature or brightness. However, such cases were so rare in the selected SAR images that this approach was discarded. To achieve bimodal distribution, the LF calculation procedure can be applied to SAR scenes divided into sub-scenes (size of approximately $1000 \times 1000$ pixels), which will demand more processing time. Such a definition of the threshold could serve as a more robust approach when developing an independent method for automatic SAR LF retrieval. For the purposes of this study, the quality of the suggested simple threshold method was considered sufficient because of the quality control steps that were undertaken. Firstly, visual inspection of every SAR LF subset was performed; it indicated that we detected most of the leads. Secondly, the ambiguous cases were excluded. And finally, the analysis was limited to only those AMSR-E LF grid cells where both data sets give a non-zero value of LF.

Analysing the results of the comparison between AMSR-E and SAR, one should keep in mind that the surface parameters that these two instruments are sensitive to are not exactly the same. The mechanisms that form the signal from an area with leads, represented by either open water or thin ice, are substantially different for SAR (sensitive to roughness) and AMSR-E (sensitive to emitted brightness). In addition, they have a different resolution: $150 \mathrm{~m} \times 150 \mathrm{~m}$ for SAR and $6 \mathrm{~km} \times 4 \mathrm{~km}$ for AMSR-E (the footprint size of the $89 \mathrm{GHz}$ channel). Thus, SAR is capable of identifying the leads in accurate locations and resolving their limits correctly (this was concluded from visual inspection of SAR retrievals, including comparison to a MODIS image). For AMSR-E the signal is an aggregated effect of all the surfaces present in the grid cell: open water, thin ice, and thicker ice, from which the percentage of open water/thin ice per grid cell needs to be deduced.

It should be noted that even an improved AMSR-E LF method would still have its limitations. For example, it would 
not be able to capture leads narrower than $3 \mathrm{~km}$ due to its resolution, while leads as narrow as a few metres transmit turbulent heat more than 2 times as efficient as the ones hundreds of metres wide (Marcq and Weiss, 2012). For studies like e.g. assessing the integrated heat fluxes through leads in wintertime, the AMSR-E LF data set alone will thus not be sufficient and other methods should be used in addition. Another limitation of such a method would be the retrieval of LF in summer, when the interpretation of passive microwave observations is challenging.

\section{Conclusions}

This work was partly motivated by the need for an accurate pan-Arctic lead fraction (LF) data set for the initialization and evaluation of regional sea ice models. One such data set was identified as having good potential for the purpose - daily pan-Arctic LF retrieved from Advanced Microwave Scanning Radiometer - Earth Observing System (AMSR-E), a passive microwave instrument independent of cloud cover and light conditions. In this study we set a goal to evaluate the AMSR-E LF data set and provide a quantitative estimate of eventual errors. These can serve as a measure of uncertainty of the product and background for a correction.

After analysis of the AMSR-E LF data set and comparison to LF retrievals from Synthetic Aperture Radar (SAR), we identified an issue with the near $100 \%$ LF values in this data set. More specifically, we concluded that the tie points used in the AMSR-E method were located too closely to each other, which caused a truncation of the real LF range. This means that LF values obtained with such tie points represent a range of values erroneously stretched over a larger range (e.g. $0-250 \%$ ) and are cut off at $100 \%$, where all the values above $100 \%$ are converted to $100 \%$, thus causing the loss of all the values above. A larger distance between the tie points would accommodate all the real LF values and give the correct range of $0-100 \%$ as output. Such an adjustment of tie points is equivalent to dividing AMSR-E LF by a certain factor. Since the information about LF $>100 \%$ is lost in the AMSR-E LF data set, we imitated the issue (peak in the LF histogram at $100 \%$ ) by multiplying SAR LF by this factor instead. In this manner we found that the current AMSR-E LF data set overestimated LF by a factor of $\sim 2-4$ over the winter 2008-2009 depending on the month considered. The absolute relative difference between the data sets expressed by $100 \times\left|\left(\overline{\mathrm{LF}_{\mathrm{AMSRE}}}-\overline{\mathrm{LF}_{\mathrm{SAR}}}\right) / \overline{\mathrm{LF}_{\mathrm{SAR}}}\right|$ decreased from $140 \%$ with no correction to $17 \%$ when using this correction factor. However, this approach is not suitable for the correction of local values, but rather reflects statistical characteristics of the data set over the whole Arctic (e.g. mean), which is confirmed by increase in the point-wise root mean square error (RMSE) between the AMSR-E LF and the SAR LF data set with correction from 33 to $43 \%$.
We argued that an adjustment of the AMSR-E LF method needed to be carried out before a more accurate error estimation could be retrieved. We therefore tried out such an adjustment by implementing the AMSR-E-based method using a higher value of the upper tie point, and found that indeed the AMSR-E LF distribution became similar to that of SAR LF. The $\mathrm{RMSE}_{h}$ used as a measure of the difference between the two histograms decreased from 5.4 to $0.9 \%$, while the point-wise RMSE for this 1-day test data set of 750 collocated LF measurements decreased from 37 to $15 \%$, or by a factor of $\sim 2$. We observed that leads were still placed in the same locations, while the LF values became lower, which corresponds to what we observed from the SAR LF data set. We estimated the new upper tie point for each month of the winter of 2008-2009 and found the values in the range from 0.103 to 0.145 , or 0.117 for the full winter as an average weighted by the number of measurements for each month. We believe that similar simple adjustment applied to the full AMSR-E LF data set will lead to significantly lower errors when evaluated using SAR, making this data set more valuable for e.g. assimilation into models or model evaluation.

Acknowledgements. The research was mainly supported by the Centre for Climate Dynamics at the Bjerknes Centre. Additional support was provided by Nansen Environmental and Remote Sensing Centre, Bergen, Norway (NERSC). Sylvain Bouillon was supported by the Research Council of Norway through the post-doc project SIMech, Sea Ice Mechanics: from satellites to numerical models (no. 231179/F20, 2014-2016). The authors would like to thank Lars Kaleschke (Institut für Meereskunde, KlimaCampus, University of Hamburg, Germany) for useful discussions and Anton Korosov (NERSC) for thorough technical support.

Edited by: C. Haas

\section{References}

ASAR Product Handbook: Issue 2.2, European Space Agency, available at: https://earth.esa.int/handbooks/asar/CNTR.html (last access: January 2014), 2007.

Beitsch, A., Kaleschke, L., and Kern, S.: Investigating HighResolution AMSR2 Sea Ice Concentrations during the February 2013 Fracture Event in the Beaufort Sea, Remote Sens., 6, 38413856, doi:10.3390/rs6053841, 2014.

Berg, A. and Eriksson, L. E. B.: SAR Algorithm for Sea Ice Concentration-Evaluation for the Baltic Sea, IEEE Geosci. Remote S., 9, 938-942, 2012.

Bouillon, S. and Rampal, P.: Presentation of the dynamical core of neXtSIM, a new sea ice model, Ocean Mod., 91, 23-37, doi:10.1016/j.ocemod.2015.04.005, 2015.

Bröhan, D. and Kaleschke, L.: A Nine-Year Climatology of Arctic Sea Ice Lead Orientation and Frequency from AMSR-E, Remote Sens., 6, 1451-1475, doi:10.3390/rs6021451, 2014.

Cavalieri, D. J.: A microwave technique for mapping thin sea ice, J. Geophys. Res., 99, 12561-12572, doi:10.1029/94JC00707, 1994. 
Farrell, S. L., Laxon, S. W., McAdoo, D. C., Yi, D., and Zwally, H. J.: Five years of Arctic sea ice freeboard measurements from the Ice, Cloud and land Elevation Satellite, J. Geophys. Res., 114, C04008, doi:10.1029/2008JC005074, 2009.

Kaleschke, L., Lupkes, C., Vihma, T., Haarpaintner, J., Bochert, A., Hartmann, J., and Heygster, G.: SSM/I sea ice remote sensing for mesoscale ocean-atmosphere interaction analysis, Can. J. Remote Sens., 27, 526-537, 2001.

Karvonen, J.: Baltic Sea ice concentration estimation based on Cband HH-Polarized SAR data, IEEE J. Sel. Top. Appl., 5, 18741884, doi:10.1109/JSTARS.2012.2209199, 2012.

Karvonen, J.: Baltic Sea ice concentration estimation based on Cband Dual-Polarized SAR data, IEEE T. Geosci. Remote, 52, 5558-5566, doi:10.1109/TGRS.2013.2290331, 2014.

Korosov, A., Hansen, M. W., and Yamakava, A.: Nansat - scientist friendly toolbox for processing satellite data, World Ocean Scientific Congress, Cochin, India, 2-8 February, TS-13/130, 2015a.

Korosov, A., Zakhvatkina, N., and Muckenhuber, S.: Ice/water classification of Sentinel-1 images, Geophysical Research Abstracts, 17, EGU2015-12487-1, EGU General Assembly 2015, Vienna, Austria, 17 April 2015b.

Korosov, A., Hansen, M. W., Yamakawa, A., Dagestad, K., Vines, A., Riechert, M., Myasoedov, A., Morozov, E. A., and Zakhvatkina, N.: Nansat v0.6.7 stable, available at: http://dx.doi.org/10. 5281/zenodo.45188, 2016.

Leigh, S., Wang, Z., and Clausi, D.: Automated Ice-Water Classification Using Dual Polarization SAR Satellite Imagery, IEEE T. Geosci. Remote, 52, 5529-5539, doi:10.1109/TGRS.2013.2290231, 2014.

Lindsay, R. W. and Rothrock, D. A.: Arctic sea ice leads from advanced very high resolution radiometer images, J. Geophys. Res., 100, 4533-4544, doi:10.1029/94JC02393, 1995.

Liu, H., Guo, H., and Zhang, L.: SVM-Based Sea Ice Classification Using Textural Features and Concentration From RADARSAT-2 Dual-Pol ScanSAR Data, IEEE J. Sel. Top. Appl, 8, 1601-1613, 2015.

Lüpkes, C., Vihma, T., Birnbaum, G., and Wacker, U.: Influence of leads in sea ice on the temperature of the atmospheric boundary layer during polar night, Geophys. Res. Lett., 35, L03805, doi:10.1029/2007GL032461, 2008.

Mäkynen, M. and Similä, M.: Thin Ice Detection in the Barents and Kara Seas With AMSR-E and SSMIS Radiometer Data, IEEE T. Geosci. Remote, IEEE Early Access Articles, 53, 5036-5053, doi:10.1109/TGRS.2015.2416393, 2015.

Marcq, S. and Weiss, J.: Influence of sea ice lead-width distribution on turbulent heat transfer between the ocean and the atmosphere, The Cryosphere, 6, 143-156, doi:10.5194/tc-6-143-2012, 2012.
Maslanik, J. A., Fowler, C., Stroeve, J., Drobot, S., Zwally, J., Yi, D., and Emery, W.: A younger, thinner Arctic ice cover: Increased potential for rapid, extensive sea-ice loss, Geophys. Res. Lett., 34, L24501, doi:10.1029/2007GL032043, 2007.

Maykut, G. A.: Energy exchange over young sea ice in the central Arctic, J. Geophys. Res., 83, 3646-3658, 1978.

Naoki, K., Ukita, J., Nishio, F., Nakayama, M., Comiso, J. C., and Gasiewski, A: Thin sea ice thickness as inferred from passive microwave and in situ observations, J. Geophys. Res., 113, 21562202, 2008.

Rampal, P., Weiss, J., and Marsan, D.: Positive trend in the mean speed and deformation rate of Arctic sea ice, 1979-2007, J. Geophys. Res., 114, C05013, doi:10.1029/2008JC005066, 2009.

Rampal, P., Bouillon, S., Ólason, E., and Morlighem, M.: neXtSIM: a new Lagrangian sea ice model, The Cryosphere Discuss., 9, 5885-5941, doi:10.5194/tcd-9-5885-2015, 2015.

Röhrs, J. and Kaleschke, L.: An algorithm to detect sea ice leads by using AMSR-E passive microwave imagery, The Cryosphere, 6 , 343-352, doi:10.5194/tc-6-343-2012, 2012.

Röhrs, J., Kaleschke, L., Bröhan, D., and Siligam, P. K.: Corrigendum to "An algorithm to detect sea ice leads by using AMSRE passive microwave imagery" published in The Cryosphere, 6, 343-352, 2012, The Cryosphere, 6, 365-365, doi:10.5194/tc-6365-2012, 2012.

Spreen, G., Kaleschke, L., and Heygster, G.: Sea ice remote sensing using AMSR-E 89-GHz channels. J. Geophys. Res.-Ocean, 113, C02S03, doi:10.1029/2005JC003384, 2008.

Svendsen, E., Matzler, C., and Grenfell, T. C.: A model for retrieving total sea ice concentration from a spaceborne dual-polarized passive microwave instrument operating near $90 \mathrm{GHz}$, Int. J. Remote Sens., 8, 1479-1487, 1987.

Weeks, W. F.: On Sea Ice, University of Alaska Press, Fairbanks, Alaska, 664 pp., 2010.

Wernecke, A. and Kaleschke, L.: Lead detection in Arctic sea ice from CryoSat-2: quality assessment, lead area fraction and width distribution, The Cryosphere, 9, 1955-1968, doi:10.5194/tc-91955-2015, 2015.

Willmes, S. and Heinemann, G.: Pan-Arctic lead detection from MODIS thermal infrared imagery, Ann. Glaciol., 56, 29-37, doi:10.3189/2015AoG69A615, 2015.

WMO: The World Meteorological Organization Sea Ice Nomenclature (WMO No. 259, TP-145, Supplement No. 5), 1989.

Zakhvatkina, N. Yu., Alexandrov, V. Yu., Johannessen, O. M., Sandven, S., and Frolov, I. Ye.: Classification of Sea Ice Types in ENVISAT Synthetic Aperture Radar Images, IEEE T. Geosci. Remote, 51, 2587-2600, doi:10.1109/TGRS.2012.2212445, 2013. 